\title{
Factores de riesgo preoperatorios en pacientes con pielonefritis xantogranulomatosa sometidos a nefrectomía
}

\author{
Preoperative risk factors in patients with xanthogranulomatous pyelonephritis undergoing \\ nephrectomy
}

\author{
Oswaldo J. Avilés-Ibarra, Efraín Maldonado-Alcaraz, Álvaro Carrasco-González, Rodrigo León-Mar, \\ Virgilio A. López-Samano, Víctor H. Rodríguez-Jasso y Jorge Moreno-Palacios* \\ Departamento de Urología, Unidad Médica de Alta Especialidad, Hospital de Especialidades, Centro Médico Nacional Siglo XXI, Instituto Mexicano \\ del Seguro Social, Ciudad de México, México
}

\begin{abstract}
Resumen
Objetivo: Identificar factores de riesgo asociados a complicaciones mayores en pacientes con pielonefritis xantogranulomatosa sometidos a nefrectomía. Método: Análisis retrospectivo de expedientes clínicos de pacientes con pielonefritis xantogranulomatosa sometidos a nefrectomía. Se analizaron el sexo, el índice de masa corporal, el índice de comorbilidad de Charlson, la clasificación del estado físico de la American Society of Anesthesiologists, la etapa de Malek, el conteo leucocitario y los valores de creatinina. Las complicaciones se estratificaron en menores y mayores según la clasificación de ClavienDindo. Se realizó análisis univariado y bivariado usando las pruebas exacta de Fisher y ji al cuadrado de Pearson, y se establecieron medidas de riesgo utilizando la odds ratio (OR). Resultados: Se analizaron 72 pacientes con una edad media de 50 años, el $83 \%$ mujeres, el $58.3 \%$ con urocultivo positivo y el $66 \%$ con litiasis renal. El 32\% tuvieron alguna complicación mayor y el 15\% requirieron admisión a la unidad de cuidados intensivos. Los valores elevados de creatinina (OR: 3.8; intervalo de confianza del 95\% [IC95\%]: 1.1-13; $p=0.02$ ) y la etapa Malek II-III (OR: 4.5; IC95\%: 1.2-17.5; $p=0.02$ ) se asociaron con complicaciones mayores. Conclusión: El estadio de Malek y los valores elevados de creatinina incrementan el riesgo de desarrollar complicaciones mayores en los pacientes con pielonefritis xantogranulomatosa sometidos a nefrectomía.
\end{abstract}

Palabras clave: Pielonefritis xantogranulomatosa. Nefrectomía. Complicaciones posoperatorias. Pronóstico.

\begin{abstract}
Objective: To identify preoperative risk factors associated with major complications in patients with xanthogranulomatous pyelonephritis undergoing total nephrectomy. Method: Retrospective analysis of patient's charts with xanthogranulomatous pyelonephritis who underwent nephrectomy. Risk factors included for analysis were gender, body mass index, Charlson comorbidity index, American Society of Anesthesiologists physical status classification, Malek's stage, leukocyte count and creatinine levels. Postoperative complications were stratified in minor and major according to Clavien-Dindo's classification. Univariate and bivariate analysis using Fisher's exact test, Pearson's chi-squared and odds ratio (OR) was performed. Results: 72 patients were analyzed, $83 \%$ women, mean age of 50 years, $58.3 \%$ positive urine cultures and $66 \%$ kidney stones. Major complications were present in $32 \%$ of cases, and $15 \%$ were admitted to the intensive care unit. Elevated creatinine
\end{abstract}

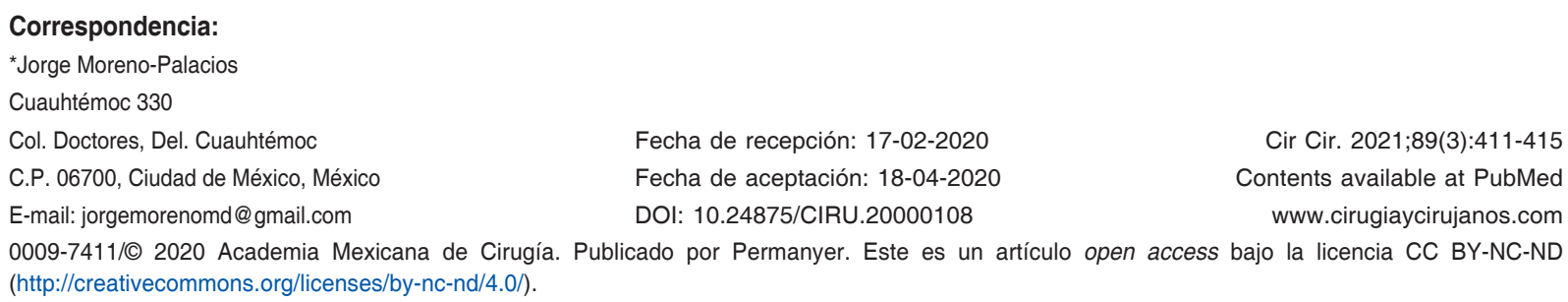

Cir Cir. 2021;89(3):411-415

Contents available at PubMed www.cirugiaycirujanos.com 0009-7411/@ 2020 Academia Mexicana de Cirugía. Publicado por Permanyer. Este es un artículo open access bajo la licencia CC BY-NC-ND (http://creativecommons.org/licenses/by-nc-nd/4.0/). 
(OR: 3.8; 95\% confidence interval [95\% Cl]: 1.1-13; $p=0.02$ ) and Malek's stage II to III (OR: 4.5; $95 \% \mathrm{Cl}: 1.2-17.5 ; p=0.02)$ were associated with major complications. Conclusion: The Malek Stage and elevated creatinine increases the risk of major complications in patients undergoing nephrectomy due to xanthogranulomatous pyelonephritis.

Key words: Xanthogranulomatous pyelonephritis. Nephrectomy. Postoperative complication. Prognosis.

\section{Introducción}

La incidencia de pielonefritis xantogranulomatosa es baja y se ha reportado que varía del $0.03 \%$ al $0.06 \%^{1,2}$. El estudio de esta patología está compuesto principalmente por reportes de casos, por lo que gran parte de la evolución natural de la enfermedad y el pronóstico de los pacientes se desconocen ${ }^{1-6}$.

Malek y Elder ${ }^{1}$ propusieron un sistema de estadificación histopatológica compuesto por tres estadios que varían dependiendo del grado de involucro renal y extrarrenal. Se ha demostrado mediante tomografía computada que es posible clasificar a los pacientes adecuadamente en uno de los tres estadios?.

La relevancia clínica de la clasificación de Malek es que ayuda a la toma de decisiones; por ejemplo, en la población pediátrica que tiene un estadio I o II se sugiere realizar una nefrectomía parcial, mientras que los pacientes con estadio III requieren un manejo con nefrectomía total. En cuanto al tipo de intervención, tanto la nefrectomía laparoscópica como la técnica abierta han demostrado resultados similares ${ }^{8-10}$.

La incidencia de complicaciones posoperatorias es alta, y de estas, el $48 \%$ se clasifican como complicaciones menores (Clavien < 3 ) y el $19 \%$ como mayores $(\text { Clavien } \geq 3)^{9}$.

El objetivo de este estudio es identificar los factores de riesgo preoperatorios relacionados con complicaciones mayores en pacientes con diagnóstico histopatológico de pielonefritis xantogranulomatosa que fueron sometidos a nefrectomía.

\section{Método}

Con previa autorización por el comité de ética se realizó un análisis retrospectivo de los expedientes clínicos de los pacientes con diagnóstico histopatológico de pielonefritis xantogranulomatosa que fueron sometidos a nefrectomía total durante el periodo de 2010 a 2016. Los expedientes clínicos incompletos fueron excluidos.

Las características basales que se incluyeron para el análisis estadístico fueron el índice de masa corporal $\geq 25 \mathrm{~kg} / \mathrm{m}^{2}$, el índice de comorbilidad de Charlson
(ICC) moderado a grave, el estado físico según la American Society of Anesthesiologists (ASA) $\geq 3$, el conteo leucocitario $\geq 12,000 \mu / \mathrm{ml}$, la anemia moderada a grave (hemoglobina $<11 \mathrm{~g} / \mathrm{dl}$ ), la creatinina $\geq$ $2.5 \mathrm{mg} / \mathrm{dl}$ y el estadio de Malek II-III como factores preoperatorios que se relacionan con un mayor riesgo de complicaciones posoperatorias. Todas las variables fueron estudiadas como dicotómicas o politómicas.

Se documentaron todas las complicaciones que ocurrieron en cada paciente, lo que quiere decir que un paciente puede presentar una o más complicaciones menores o mayores. Para el análisis estadístico solo se consideró la complicación más grave. Las complicaciones posoperatorias se estratificaron, de acuerdo con la clasificación de Clavien-Dindo, en menores y mayores ${ }^{11}$.

Para el análisis descriptivo de las variables cuantitativas se utilizaron la media ( \pm desviación estándar) y la mediana (percentiles 25-75), según el tipo de distribución. Las variables cualitativas se describieron utilizando medidas de frecuencia y proporciones.

Para determinar las diferencias estadísticamente significativas entre el grupo expuesto y no expuesto se utilizaron la prueba de ji al cuadrado de Pearson y la prueba exacta de Fisher. Se consideró como estadísticamente significativo un valor de $p<0.05$. Para establecer la magnitud y la dirección de las asociaciones se calculó la odds ratio (OR) con un intervalo de confianza del 95\% (IC95\%). Se utilizó el paquete SPSS versión 25 (IBM Chicago, II, USA).

\section{Resultados}

Se incluyeron 72 pacientes, de los cuales el $83 \%$ fueron mujeres. La edad media fue de 50 años $( \pm 14.5)$. El 58.3\% tuvieron urocultivos positivos; de estos, el $50 \%$ (21/42) fueron positivos para microorganismos productores de betalactamasas de espectro extendido. Se documentó que el $66.6 \%$ de los pacientes cursaban con algún grado de anemia; la distribución por sexo fue de 41/60 (68.3\%) y $7 / 12$ $(58.3 \%)$ para las mujeres y los hombres, respectivamente. Las características demográficas se presentan en la tabla 1. 
Tabla 1. Características demográficas

\begin{tabular}{|c|c|}
\hline Variable & $n=72$ \\
\hline Edad, media (DE) & $50 \pm 14.5$ \\
\hline Mujeres, n (\%) & $60(83.3)$ \\
\hline Diabetes mellitus, n (\%) & $26(31.9)$ \\
\hline Litiasis renal, n (\%) & $48(66)$ \\
\hline \multicolumn{2}{|l|}{ Estado físico ASA, n (\%) } \\
\hline 1 & $12(16.6)$ \\
\hline 2 & $20(27.7)$ \\
\hline 3 & $29(40.3)$ \\
\hline 4 & $11(15.4)$ \\
\hline \multirow{2}{*}{\multicolumn{2}{|c|}{$\begin{array}{l}\text { Índice de comorbilidad de } \\
\text { Charlson, n (\%) }\end{array}$}} \\
\hline & \\
\hline Leve & $56(78)$ \\
\hline Moderado & $8(11)$ \\
\hline Grave & $8(11)$ \\
\hline \multicolumn{2}{|l|}{ IMC, n (\%) } \\
\hline$<25 \mathrm{~kg} / \mathrm{m}^{2}$ & $31(43)$ \\
\hline $25-29,9 \mathrm{~kg} / \mathrm{m}^{2}$ & $22(30.6)$ \\
\hline$\geq 30 \mathrm{~kg} / \mathrm{m}^{2}$ & $19(26.4)$ \\
\hline \multicolumn{2}{|l|}{ Análisis general de orina } \\
\hline Resultados anormales & $61(84.7)$ \\
\hline Nitritos positivos & $22(30.6)$ \\
\hline Esterasa leucocitaria & $53(73.6)$ \\
\hline Piuria & $60(83.3)$ \\
\hline Eritrocitos & $51(70.8)$ \\
\hline Cultivo de orina positivo & $42(58.3)$ \\
\hline Microorganismos BLEE & $21(29.2)$ \\
\hline Enfermedad renal crónica, n (\%) & $12(16.6)$ \\
\hline Anemia en mujeres & $41(68.3)$ \\
\hline Leve & $9(15.1)$ \\
\hline Moderada & $22(36.6)$ \\
\hline Grave & $10(16.6)$ \\
\hline Anemia en hombres & $7(58.3)$ \\
\hline Leve & $3(25)$ \\
\hline Moderada & $3(25)$ \\
\hline Grave & $1(8.6)$ \\
\hline Leucocitos $\geq 12,000 \mu / \mathrm{ml}, \mathrm{n}(\%)$ & $31(43.1)$ \\
\hline Creatinina $\geq 2.5 \mathrm{mg} / \mathrm{dl}, \mathrm{n}(\%)$ & $12(16.7)$ \\
\hline \multicolumn{2}{|l|}{ Lateralidad, n (\%) } \\
\hline Izquierdo, n (\%) & $42(68.3)$ \\
\hline Derecho, n (\%) & $30(41.7)$ \\
\hline \multicolumn{2}{|l|}{ Estadio Malek* } \\
\hline I & $22(30.5)$ \\
\hline$\|$ & $26(36.2)$ \\
\hline III & $24(33.3)$ \\
\hline
\end{tabular}

ASA: American Society of Anesthesiologists; BLEE: betalactamasas de espectro extendido; DE: desviación estándar; IMC: índice de masa corporal.

*El estadio de Malek se estableció por medio de tomografía computada.

La prevalencia global de complicaciones, tanto intraoperatorias como posoperatorias, fue del $92 \%$. Se documentaron seis complicaciones intraoperatorias:
Tabla 2. Complicaciones de acuerdo con la clasificación de Clavien-Dindo

\begin{tabular}{llc}
\hline \multicolumn{2}{l}{ Complicaciones menores } & $\mathbf{n}(\%)$ \\
\hline II & Fiebre & $9(13)$ \\
\multicolumn{1}{l}{ Complicaciones } & mayores & $29(40)$ \\
\hline IIIA & Neumotórax & \\
IIIB & Absceso en sitio quirúrgico & $1(1)$ \\
& Hematoma retroperitoneal & $4(6)$ \\
IVA & Fístula enterocutánea & $3(4)$ \\
IVB & Disfunción orgánica & $1(1)$ \\
& Choque séptico con falla orgánica & $7(10)$ \\
V & múltiple & $4(6)$ \\
& Infarto agudo de miocardio & $2(3)$ \\
& Choque séptico &
\end{tabular}

tres lesiones vasculares y tres perforaciones de colon.

De acuerdo con la clasificación de Clavien-Dindo, la incidencia de complicaciones menores y mayores fue del $53 \%$ y el $35 \%$, respectivamente. El $40 \%$ de los casos requirió transfusión de al menos un paquete globular; de estos, el $72 \%$ (21/29) requirieron dos o más paquetes globulares.

La mayor parte de las complicaciones clasificadas como Clavien 3 fueron a causa de ingreso en la unidad de cuidados intensivos (UCI). Todas las complicaciones Clavien 4B fueron secundarias a choque séptico con falla orgánica múltiple. El resto de las complicaciones se presentan en la tabla 2.

Posterior al análisis bivariado, los valores de creatinina $\geq 2.5 \mathrm{mg} / \mathrm{dl}$ (OR: 3.8; IC95\%: 1.1-13; $\mathrm{p}=0.02$ ) y el estadio Malek II-III (OR: 4.5; IC95\%: 1.2-17.5; $p=0.02$ ) se asociaron con un mayor riesgo de complicaciones posoperatorias. Se encontró que existe una tendencia entre el conteo leucocitario $\geq 12,000$ $\mu / \mathrm{ml}$ y el ICC moderado a grave en relación con el desarrollo de complicaciones mayores (Tabla 3 ).

\section{Discusión}

Según nuestro conocimiento, este estudio representa la cohorte más grande que se ha reportado en la literatura y es el primero en identificar factores pronósticos en pacientes con pielonefritis xantogranulomatosa.

La prevalencia de pielonefritis xantogranulomatosa en las mujeres fue tres veces mayor que en los hombres, con una relación de 4:1, lo cual es similar a lo reportado en otras series ${ }^{2,5,10}$. 
Tabla 3. Análisis bivariado: factores pronósticos asociados con complicaciones mayores

\begin{tabular}{|c|c|c|c|c|}
\hline Variable & $\begin{array}{l}\text { Complicaciones } \\
\text { menores } \\
(n=49)\end{array}$ & $\begin{array}{l}\text { Complicaciones } \\
\text { mayores } \\
(n=23)\end{array}$ & $\begin{array}{c}\text { OR } \\
(\mathrm{IC} 95 \%)\end{array}$ & $p$ \\
\hline \multicolumn{5}{|l|}{ Sexo } \\
\hline Femenino & $9(75)$ & $3(25)$ & 1 & \\
\hline Masculino & $40(66.7)$ & 20 (33.3) & $1.5(0.3-6.1)$ & \\
\hline \multicolumn{5}{|l|}{ IMC } \\
\hline$<25 \mathrm{~kg} / \mathrm{m}^{2}(\%)$ & $21(67.7)$ & $10(32.3)$ & 1 & \\
\hline$\geq 25 \mathrm{~kg} / \mathrm{m}^{2}(\%)$ & $28(68.2)$ & $13(31.8)$ & $0.9(0.3-2.6)$ & $0.5^{\dagger}$ \\
\hline \multicolumn{5}{|l|}{ ICC } \\
\hline Leve (\%) & $41(73.2)$ & $15(26.8)$ & 1 & \\
\hline $\begin{array}{l}\text { Moderado } \\
\text {-grave \%) }\end{array}$ & $8(50)$ & $8(50)$ & $2.7(0.8-8.5)$ & $0.07^{*}$ \\
\hline \multirow{2}{*}{\multicolumn{5}{|c|}{$\begin{array}{l}\text { Estado físico } \\
\text { ASA }\end{array}$}} \\
\hline & & & & \\
\hline$\leq 2(\%)$ & $24(77.4)$ & $7(22.6)$ & 1 & \\
\hline$\geq 3(\%)$ & $25(61)$ & $16(39)$ & $2.1(0.7-6.2)$ & $0.1^{\dagger}$ \\
\hline \multirow{2}{*}{\multicolumn{5}{|c|}{$\begin{array}{l}\text { Leucocitos } \\
\leq 12,000 \mu / \mathrm{ml}\end{array}$}} \\
\hline & & & & \\
\hline Presente (\%) & $31(75.6)$ & $10(24.4)$ & 1 & \\
\hline Ausente (\%) & $18(58)$ & $13(42)$ & $2.2(0.8-6.1)$ & $0.09^{\dagger}$ \\
\hline \multicolumn{5}{|l|}{ Hemoglobina } \\
\hline \multicolumn{5}{|l|}{$<11 \mathrm{~g} / \mathrm{dl}$} \\
\hline Presente (\%) & $23(63.9)$ & $13(36.1)$ & 1 & \\
\hline Ausente (\%) & $36(72.2)$ & $10(27.8)$ & $1.4(0.5-3.9)$ & $0.4^{+}$ \\
\hline \multicolumn{5}{|l|}{ Creatinina } \\
\hline Presente (\%) & $5(41.6)$ & $7(58.4)$ & 1 & \\
\hline Ausente (\%) & $44(66.6)$ & $16(33.3)$ & $3.8(1.06-13.8)$ & 8) $0.03^{*}$ \\
\hline \multicolumn{5}{|l|}{$\begin{array}{l}\text { Estadio de } \\
\text { Malek }\end{array}$} \\
\hline I (\%) & $20(87)$ & $3(13)$ & 1 & \\
\hline II-III (\%) & $29(59.2)$ & $20(40.8)$ & $4.5(1.2-17.5)$ & ) $0.02^{+}$ \\
\hline
\end{tabular}

La prevalencia de pacientes con diabetes mellitus fue menor en nuestra población ${ }^{12,13}$. Sin embargo, continuó siendo la comorbilidad más frecuente, presentándose en tres de cada diez casos. Consideramos que, tal como se ha descrito en la literatura, la diabetes mellitus desempeña un papel determinante en el desarrollo de pielonefritis xantogranulomatosa, aunque es necesario disponer de series más grandes para establecer una asociación estadística.

De todos los urocultivos positivos de nuestra serie, en más del $50 \%$ se desarrolló una bacteria productora de betalactamasas de espectro extendido. Aunque no contamos con los patrones de crecimiento específicos, se ha reportado que más del $50 \%$ de los pacientes con pielonefritis xantogranulomatosa tienen un urocultivo positivo previo a la cirugía, $a^{1,2,6,14}$. Este hallazgo podría relacionarse con una antibioticoterapia inadecuada para el tratamiento de infecciones urinarias recurrentes, las cuales son comunes en estos pacientes ${ }^{1,6,9}$.

La mayor parte de las complicaciones menores estuvieron conformadas por transfusiones de paquetes globulares. La elevada tasa de hemotransfusiones encontrada en nuestra serie puede deberse, en parte, a la alta prevalencia preoperatoria de anemia moderada y grave. No estuvo disponible para el análisis la información respecto al sangrado transoperatorio, pero Shah, et al. ${ }^{9}$ reportan una media de sangrado de $257 \mathrm{ml}$ en pacientes sometidos a nefrectomía abierta. Consideramos que, aunque este valor no representa un sangrado clínicamente significativo, podría reducirse mediante el abordaje laparoscópico.

La única serie reportada en la literatura que utiliza la clasificación de Clavien-Dindo es la de Shah, et al. ${ }^{9}$, en la que se halló una incidencia de complicaciones mayores del $13.5 \%$, la cual es menor que en nuestra serie. Esta diferencia puede atribuirse a una mayor prevalencia de comorbilidad en nuestra población, tal como demuestra la tendencia encontrada entre el ICC y el riesgo de complicaciones mayores.

La mayor parte de las complicaciones mayores estuvieron conformadas por admisiones a la UCI. Entre las indicaciones de ingreso, las más comunes fueron falla de un solo órgano y choque séptico. Estos hallazgos sugieren que retrasar la intervención quirúrgica hasta optimizar los parámetros clínicos (anemia, resolución de la infección del tracto urinario, función renal, etc.), así como realizar una valoración preoperatoria por la $\mathrm{UCl}$, pueden ser conductas factibles para mejorar el pronóstico.

Se encontró que los pacientes con valores de creatinina $\geq 2.5 \mathrm{mg} / \mathrm{dl}$ tienen tres veces más riesgo de complicaciones mayores; no obstante, los pacientes con estadio Malek II-III tienen un riesgo aún mayor. Los valores de creatinina son modificables, pero no es el mismo escenario con el estadio de Malek.

La mayor parte de las limitaciones de este estudio se relacionan con la baja incidencia de esta enfermedad, lo cual dificulta el reclutamiento de una muestra más representativa que permita realizar un análisis multivariado y ajustar por una mayor cantidad de factores de confusión. Otra limitante importante es el diseño metodológico.

Debido a que se trata de una enfermedad poco frecuente y con unas tasas de comorbilidad y de 
complicaciones posoperatorias elevadas, consideramos que es relevante desarrollar estudios prospectivos y multicéntricos que permitan esclarecer el pronóstico de estos pacientes.

\section{Conclusiones}

El estadio de Malek y los valores elevados de creatinina incrementan el riesgo de desarrollar complicaciones mayores en los pacientes con pielonefritis xantogranulomatosa sometidos a nefrectomía.

\section{Agradecimientos}

Los autores agradecen al Instituto Mexicano del Seguro Social y a todos los participantes que por su dedicación y trabajo permitieron desarrollar este trabajo de investigación.

\section{Conflicto de intereses}

Todos los autores declaran que no existe ningún tipo de conflicto de intereses.

\section{Responsabilidades éticas}

Protección de personas y animales. Los autores declaran que para esta investigación no se han realizado experimentos en seres humanos ni en animales.

Confidencialidad de los datos. Los autores declaran que han seguido los protocolos de su centro de trabajo sobre la publicación de datos de pacientes.
Derecho a la privacidad y consentimiento informado. Los autores han obtenido el consentimiento informado de los pacientes y/o sujetos referidos en el artículo. Este documento obra en poder del autor de correspondencia.

\section{Bibliografía}

1. Malek RS, Elder JS. Xanthogranulomatous pyelonephritis: a critical analysis of 26 cases and of the literature. J Urol. 1978;119:589-93.

2. Kuo C-C, Wu C-F, Huang C-C, Lee Y-J, Lin W-C, Tsai C-W, et al. Xanthogranulomatous pyelonephritis: critical analysis of 30 patients. Int Urol Nephrol. 2011;43:15-22.

3. AIDarrab R, AlAkrash H, AlKhateeb S, Al Bqami N. A case report of a xanthogranulomatous pyelonephritis case mimicking the recurrence of renal cell carcinoma after partial nephrectomy. Urol Ann. 2015;7:524-6.

4. Parsons MA, Harris SC, Longstaff AJ, Grainger RG. Xanthogranulomatous pyelonephritis: a pathological, clinical and aetiological analysis of 87 cases. Diagn Histopathol. 1983;6:203-19.

5. Korkes F, Favoretto RL, Bróglio M, Silva CA, Castro MG, Pérez MDC. Xanthogranulomatous pyelonephritis: clinical experience with 41 cases. Urology. 2008;71:178-80

6. Siddappa S, Ramprasad K, Muddegowda MK. Xanthogranulomatous pyelonephritis: a retrospective review of 16 cases. Korean J Urol. 2011;52:421-4.

7. Goldman SM, Hartman DS, Fishman EK, Finizio JP, Gatewood OM, Siegelman SS. CT of xanthogranulomatous pyelonephritis: radiologic-pathologic correlation. Am J Roentgenol. 1984;142:963-9.

8. Arvind NK, Singh O, Ali Q, Gupta SS, Sahay S. Laparoscopic nephrectomy in xanthogranulomatous pyelonephritis: 7-year single-surgeon outcome. Urology. 2011;78:797-801.

9. Shah KJ, Ganpule AP, Kurien A, Muthu V, Sabnis RB, Desai MR. Laparoscopic versus open nephrectomy for xanthogranulomatous pyelonephritis: an outcome analysis. Indian J Urol. 2011;27:470-4

10. Vanderbrink BA, Ost MC, Rastinehad A, Anderson A, Badlani GH, Smith $A D$, et al. Laparoscopic versus open radical nephrectomy for xanthogranulomatous pyelonephritis: contemporary outcomes analysis. J Endourol. 2007;21:65-70.

11. Dindo D, Demartines N, Clavien PA. Classification of surgical complications: a new proposal with evaluation in a cohort of 6336 patients and results of a survey. Ann Surg. 2004;240:205-13.

12. Caliskan S, Ozsoy E, Kaba S, Koca O, Ozturk MI. Xanthogranulomatous pyelonephritis. Arch Iran Med. 2016;19:712-4

13. Al-Ghazo MA, Ghalayini IF, Matalka II, Al-Kaisi NS, Khader YS. Xanthogranulomatous pyelonephritis: analysis of 18 cases. Asian J Surg. 2006;29:257-61.

14. Korkes F, Favoretto RL, Bróglio M, Silva CA, Castro MG, Pérez MDC Xanthogranulomatous pyelonephritis: clinical experience with 41 cases. Urology. 2008;71:178-80. 